RELATO DE CASO

\title{
Duplo arco aórtico: a quebra do silêncio
}

\section{Double aortic arch: the break of silence}

\author{
Ana Rita Abrão', Vanessa Queiroz de Souza', Eduardo de Oliveira Campos², Rosangeles Konrad Brito ${ }^{3}$, Alexandre Dias Mançano ${ }^{4}$
}

\begin{abstract}
Resumo
Anéis vasculares representam 1-2\% dos casos das cardiopatias congênitas. Relatamos um caso raro de duplo arco aórtico. Mulher, 60 anos, procurou atendimento na clínica médica apresentando 1 ano de história de disfagia, 6 meses de dispneia e 2 meses de dor torácica esporádica. Raio X de tórax revelou: hiperinsuflação pulmonar difusa, alargamento mediastinal, coração com volume e configurações normais, arco aórtico à direita e alterações degenerativas vertebrais. Tomografia computadorizada do tórax: arco aórtico duplo circundando e comprimindo a traqueia e o esôfago. Arco direito mais calibroso, emergindo dele o tronco braquiocefálico. Do arco esquerdo emergem a artéria carótida comum e a subclávia esquerda. Diagnóstico: anel vascular traqueoesofagiano decorrente do duplo arco aórtico, sendo o arco direito dominante. No presente caso, optou-se por seguimento clínico da paciente, levando-se em conta a intensidade dos sintomas apresentados.
\end{abstract}

Descritores: Malformações vasculares; anormalidades congênitas; cardiopatias congênitas; aorta torácica.

\begin{abstract}
Vascular rings represent 1 to $2 \%$ of cases of congenital heart disease. We report a rare case of double aortic arch. A 60-year-old woman was admitted to the hospital presenting a one-year history of dysphagia, six months of dyspnea and two months of sporadic chest pain. Radiograph of the chest revealed diffuse pulmonary hyper inflation, widening of the mediastinum, heart of normal size and shape, a right-sized aortic arch, and degenerative changes of the thoracic spine. Computed tomography of the chest showed a double aortic arch encircling and compressing the trachea and the esophagus. The right aortic arch had a larger caliber, with brachiocephalic trunk arising from it. The left common carotid artery and the left subclavian artery arose from the left aortic arch. Diagnosis: tracheoesophageal vascular ring due to double aortic arch, with dominant right arch. In this case, we chose to follow the patient medically, taking into consideration the mildness of the symptoms.
\end{abstract}

Keywords: Vascular malformations; congenital abnormalities; heart defects, congenital; aorta, thoracic.

\section{Introdução}

Anéis vasculares são anormalidades do arco aórtico que envolvem parcialmente ou completamente o esôfago e/ ou a traqueia, podendo causar disfagia e/ou sintomas respiratórios $^{1-5}$. São mais encontrados em crianças devido ao aparecimento precoce dos sintomas, e a doença nos adultos é rara. Contudo, pode ser silenciosa e descoberta ao acaso - ou o despertar do quadro clínico ocorre com sintomas exuberantes.

Importante causa de obstrução traqueal, os anéis vasculares foram classificados pelo International Congenital Heart Surgery Nomenclature and Database Committee (Tabela 1) $)^{6,7}$. Descrevemos um caso de duplo arco aórtico com arco direito dominante, em uma mulher com 60 anos, cujos sintomas despertaram há 1 ano.

Anéis vasculares representam 1-2\% dos casos das cardiopatias congênitas². O objetivo deste trabalho foi relatar um caso raro de duplo arco aórtico.

\section{Relato do caso clínico}

Mulher, 60 anos, procurou o atendimento na unidade de clínica médica em janeiro de 2009, apresentando 1 ano de história de disfagia, 6 meses de dispneia aos moderados esforços e 2 meses de dor torácica esporádica: dor retroesternal, com leve intensidade, em pontada e com irradiação para o dorso.

\footnotetext{
${ }^{1}$ Médica Residente do Departamento de Radiologia e Diagnóstico por Imagem do Hospital Regional de Taguatinga (HRT) da Secretaria de Estado de Saúde do Distrito Federal, Brasília (DF) Brasil.

${ }^{2}$ Médico Residente do Departamento de Clínica Médica do HRT da Secretaria de Estado de Saúde do Distrito Federal, Brasília (DF), Brasil.

${ }^{3}$ Médica Cardiologista do Departamento de Cardiologia do HRT da Secretaria de Estado de Saúde do Distrito Federal, Brasília (DF), Brasil.

${ }^{4}$ Coordenador da Residência Médica de Radiologia e Diagnóstico por Imagem do HRT da Secretaria de Estado de Saúde do Distrito Federal, Brasília (DF), Brasil.

Não foram declarados conflitos de interesse associados à publicação deste artigo.

Submetido em: 28.6.09. Aceito em: 13.12.10

J Vasc Bras. 2011;10(1):59-63.
} 
Tabela 1 - Classificação dos anéis vasculares definidos pelo International Congenital Heart Surgery Nomenclature and Database Committee $^{6,7}$

\section{Duplo arco aórtico \\ Arco aórtico direito dominante \\ Arco aórtico esquerdo dominante \\ Arcos balanceados}

Arco aórtico direito - ligamento esquerdo

Imagem em espelho dos ramos arteriais

Artéria subclávia esquerda retroesofágea

Aorta circunflexa

Compressão da artéria inominada

Compressão da artéria pulmonar

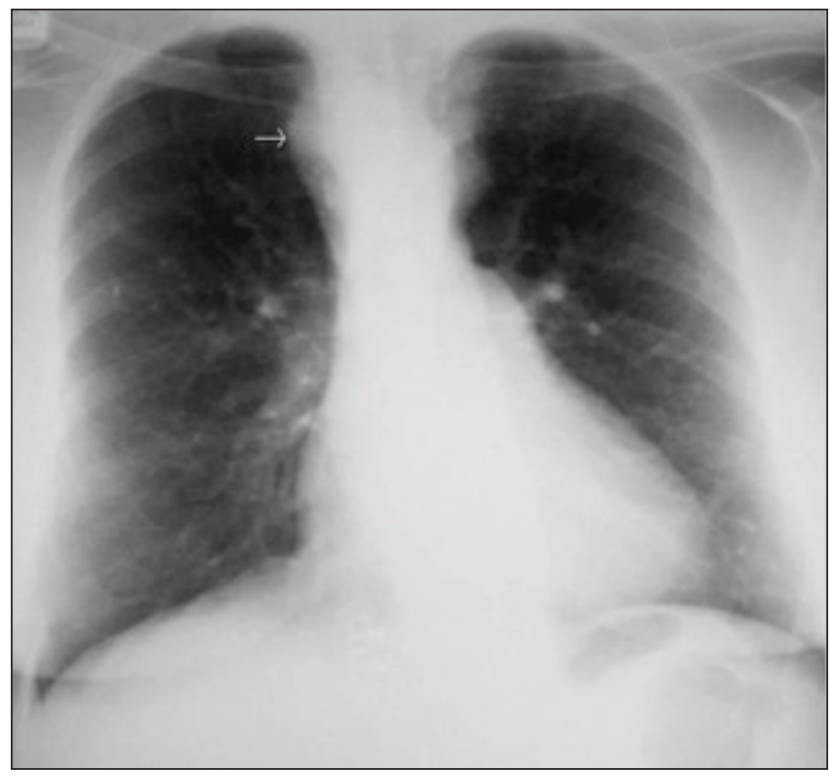

Figura 1 - Raio X de tórax posteroanterior. Sinais de hiperinsuflação pulmonar difusa e bilateral. Alargamento mediastinal. Coração com volume e configurações normais. Arco aórtico à direita (seta branca)

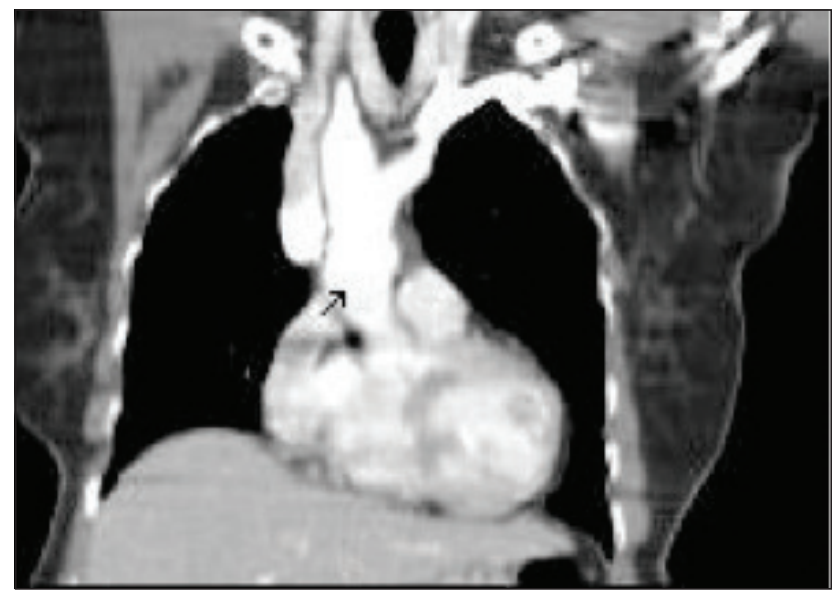

Figura 2 - Tomografia computadorizada de tórax. Reformatação coronal com contraste endovenoso. Aorta emergindo do ventrículo esquerdo (seta), emitindo um arco à direita, mais calibroso, e outro arco à esquerda
O exame clínico da paciente não apresentou anormalidades. Prosseguiu-se à investigação com métodos de imagem.

O eletrocardiograma realizado no momento da consulta não apresentou alterações.

A radiografia simples de tórax revelou sinais de hiperinsuflação pulmonar difusa e bilateral, alargamento mediastinal, coração com volume e configurações normais, arco aórtico à direita e alterações degenerativas vertebrais (Figura 1).

A tomografia computadorizada (TC) de tórax demonstrou arco aórtico duplo circundando a traqueia e o esôfago, com compressão e deslocamento dessas estruturas (Figura 2). O arco aórtico direito era o mais calibroso (Figuras 3 e

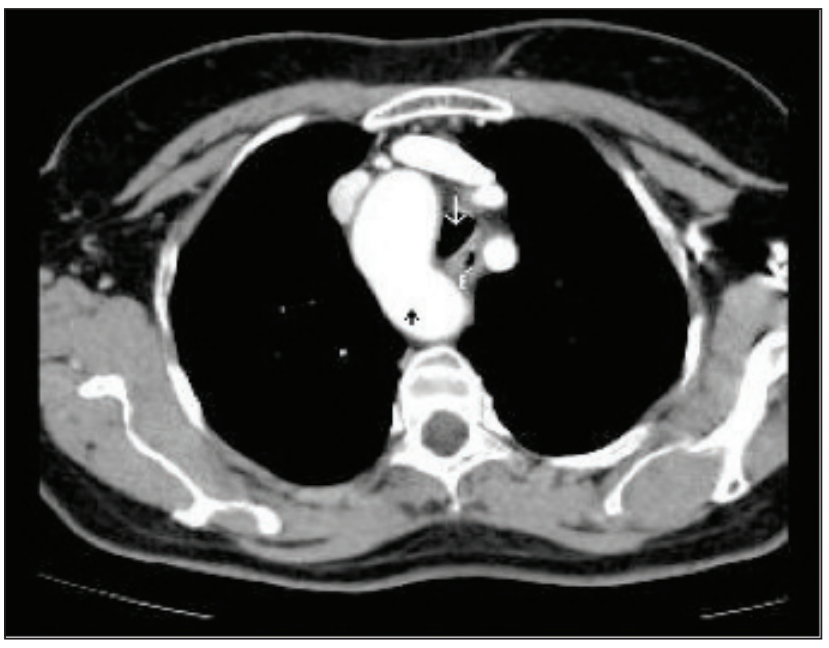

Figura 3 - Tomografia computadorizada de tórax axial com contraste endovenoso, janela para mediastino. Arco aórtico direito (seta preta) envolvendo a traqueia (seta branca). E: esôfago

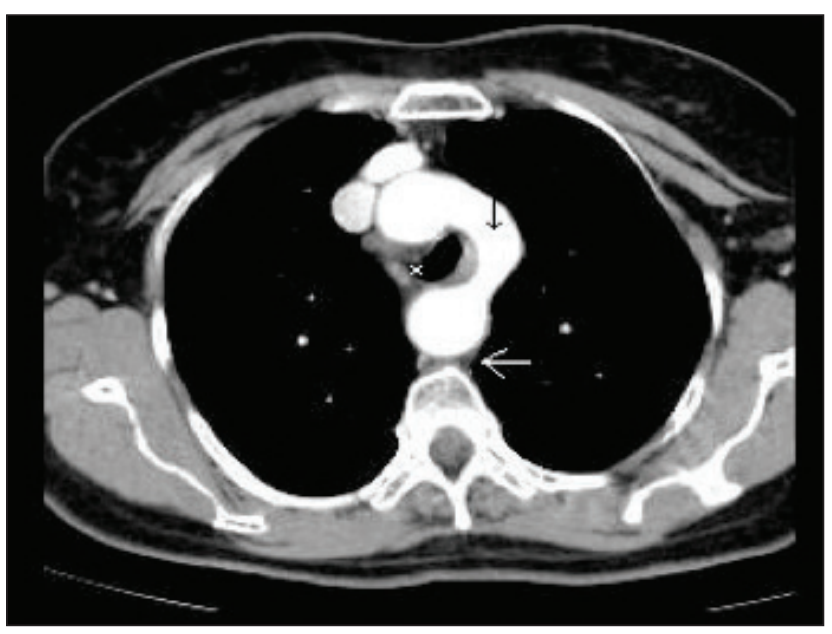

Figura 4 - Tomografia computadorizada de tórax axial com contraste endovenoso, janela para mediastino. Arco aórtico esquerdo (seta preta) envolvendo a traqueia $(x)$ e comprimindo o esôfago (seta branca) 
4), dominante, do qual emergiam a artéria carótida e a artéria subclávia ipsilaterais. Do arco aórtico esquerdo emergiam a artéria carótida comum e a artéria subclávia esquerda (Figura 5). A aorta descendente encontrava-se à direita da linha média. O diagnóstico foi anel vascular traqueoesofagiano decorrente de duplo arco aórtico com o arco direito dominante (Figura 6).

O cateterismo cardíaco evidenciou circulação coronária sem lesões obstrutivas e confirmou o achado da TC ao mostrar a presença do duplo arco aórtico (Figura 7).

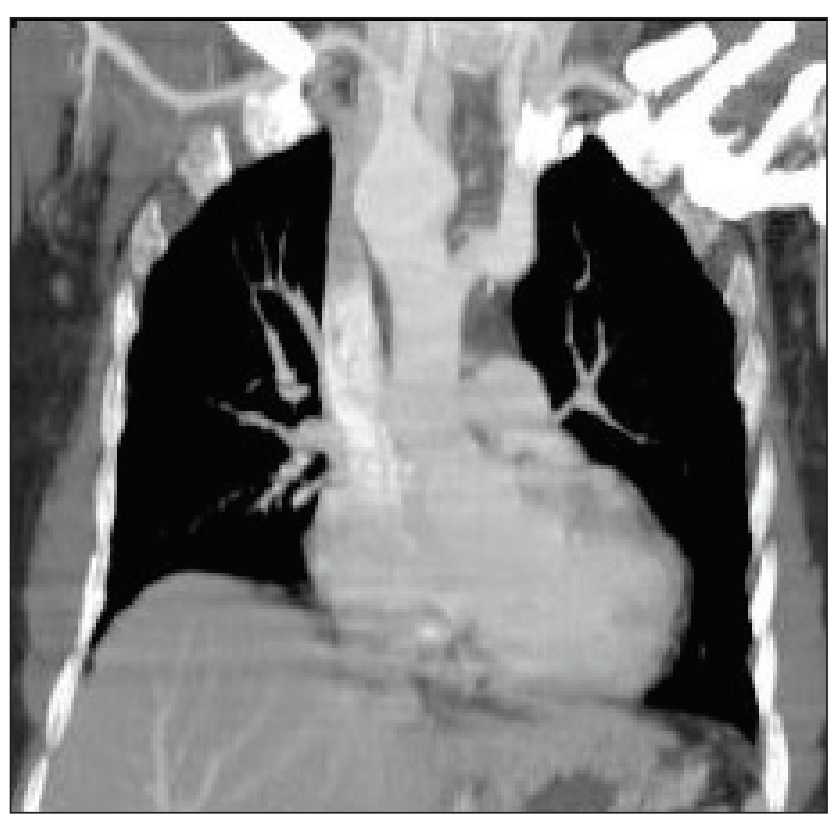

Figura 5 - Tomografia computadorizada de tórax com reformatação coronal e contraste endovenoso. Arco aórtico direito, mais calibroso, dominante, do qual emerge o tronco braquiocefálico. Arco aórtico esquerdo com seus ramos: artéria carótida comum e artéria subclávia esquerda

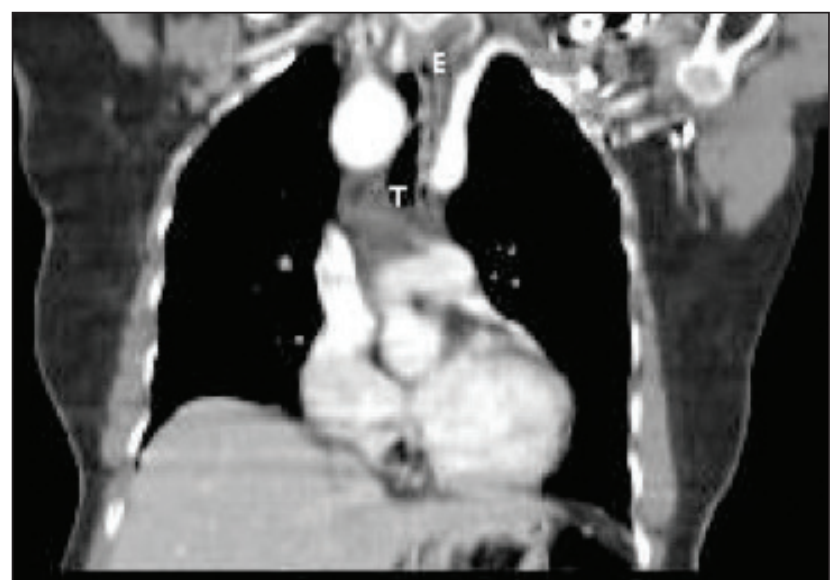

Figura 6 - Tomografia computadorizada de tórax com reformatação coronal e contraste endovenoso. Anel vascular comprimindo o esôfago (E) e a traqueia ( $\mathrm{T}$ )
A paciente encontra-se, no presente momento, em bom estado geral, porém sintomática. Foi avaliada na unidade de cirurgia cardíaca e, diante dos exames e da intensidade dos sintomas, optou-se por conduta clínica e seguimento ambulatorial.

\section{Discussão}

O desenvolvimento embriológico vascular cursa com modificações nos seis pares de arcos aórticos que estão conectados a duas aortas primitivas, a ventral e a dorsal (Figura 8$)^{8}$. A maior porção do primeiro, segundo e quinto arco sofre regressão. O terceiro arco forma as artérias carótidas. O ramo do botão ventral do sexto arco encontra-se

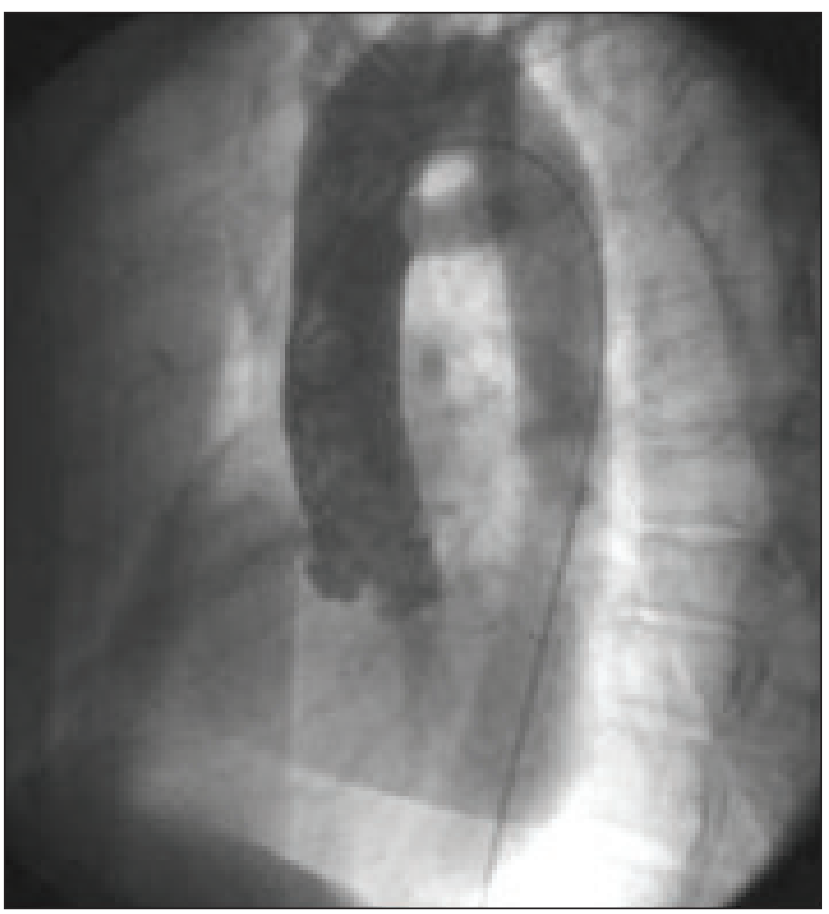

Figura 7 - Cinecoronariografia: anel vascular devido ao duplo arco aórtico

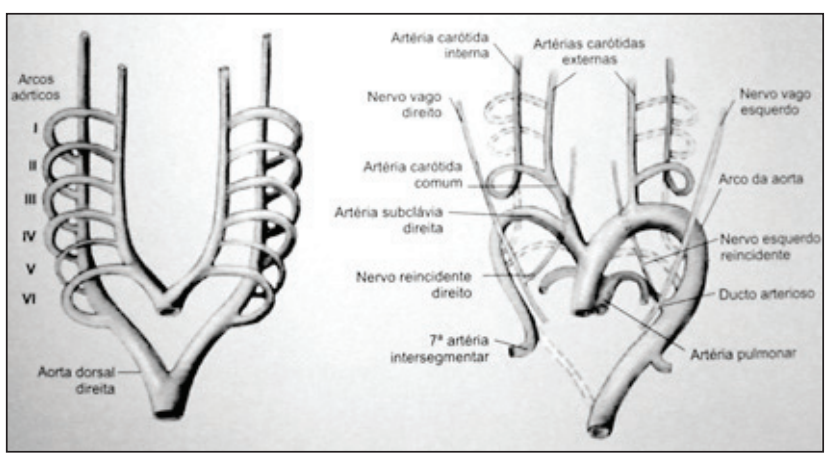

Fonte: Mafeei FHA, Lastoria S, Yoshida WB, et al. ${ }^{8}$

Figura 8 - Diagrama dos arcos aórticos. Seis pares de arcos desenvolvemse entre a aorta dorsal e a ventral 
com o botão pulmonar para formar a artéria pulmonar. Do lado direito, a contribuição dorsal do sexto arco desaparece; do lado esquerdo, persiste como ducto arterioso. As sete artérias intersegmentares surgem do ramo dorsal da aorta e formam as artérias subclávias ${ }^{3,8,9}$.

Normalmente, a porção direita do quarto arco regride, deixando o conhecido arco aórtico esquerdo. Se persistirem os ramos direito e esquerdo do quarto arco, forma-se o duplo arco aórtico ${ }^{3,8}$.

$\mathrm{O}$ anel vascular formado pelo duplo arco aórtico foi descrito primeiramente por Hommell, em 1737. Contudo, o termo anel vascular foi difundido apenas em 1945, quando Robert Gross dissecou com sucesso a divisão do anel, um duplo arco aórtico ${ }^{1}$. A incidência do anel vascular é menor que $0,2 \%$ anualmente, sendo primeiramente diagnosticado em crianças, cujos sintomas respiratórios e gastrointestinais representam, respectivamente, 93 e $41 \%{ }^{1}$. Relatamos o caso de uma paciente idosa, 60 anos, que apresentou uma história clínica com 1 ano de evolução de disfagia e dispneia. Disfagia é o sintoma mais comum nos casos de anéis vasculares em

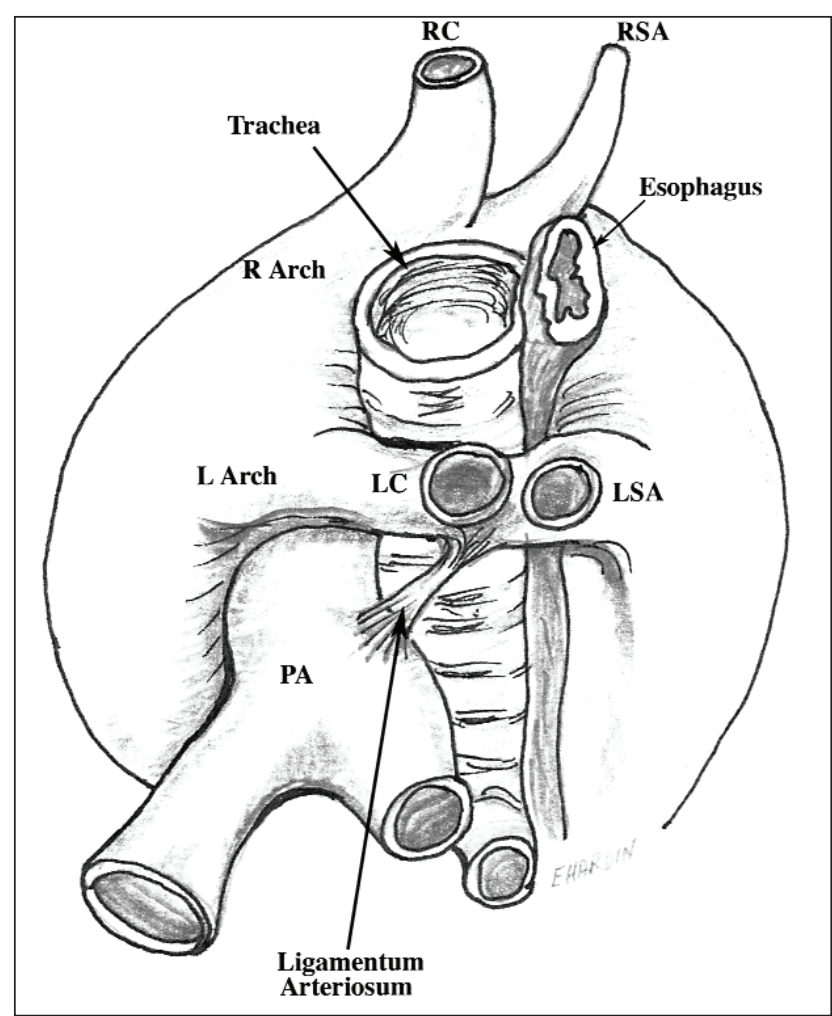

Fonte: Hardin, Brevetti, Sanusi, et al. ${ }^{1}$

Figura 9 - Visão lateral esquerda. Ilustração do duplo arco aórtico. RC: artéria carótida direita; RSA: artéria subclávia direita; R Arch: arco direito; L Arch: arco esquerdo; LC: artéria carótida esquerda; LSA: artéria subclávia esquerda; PA: artéria pulmonar. adultos $^{1}$. Os mecanismos que possibilitam aos pacientes se manterem assintomáticos ainda permanecem obscuros.

O anel vascular formado pelo clássico duplo arco aórtico ocorre da seguinte maneira: a aorta ascendente cursa anterior à traqueia e divide-se em dois arcos. Os arcos ramificam-se nas artérias carótidas e subclávias ipsilaterais, passando sobre os respectivos brônquios, e se encontram no lado oposto, atrás do esôfago, formando a aorta descendente (Figura 9).

O duplo arco aórtico é um clássico exemplo de anel vascular - o arco é formado pela própria aorta. O fato de o ducto arterioso correr entre a aorta e o sistema arterial pulmonar é um fator agravante adicional de compressão da parede anterior da traqueia. A compressão esofagiana ocorre através do segmento descendente da aorta ${ }^{4,8-10}$.

Dentre os casos de duplo arco aórtico, o arco direito é o dominante em $75 \%$, sendo o arco esquerdo patente na maioria das vezes. Quando o arco esquerdo encontra-se atrésico, a atresia é geralmente distal à subclávia esquerda. Nos casos em que o arco aórtico esquerdo é o dominante, o direito geralmente é patente. De forma geral, apenas 30\% dos arcos que não são dominantes são atrésicos ${ }^{4,8}$.

No caso apresentado, os arcos são suficientemente amplos para perfundir os suplementos arteriais, sendo o arco aórtico direito mais calibroso. A compressão da traqueia é ocasionada pelo arco aórtico direito e a compressão esofagiana, pela aorta descendente.

Sintomas respiratórios aparecem devido à compressão da traqueia pela dilatação da aorta, que pode ocorrer por mecanismos estáticos ou dinâmicos. A administração de fluidos e o exercício podem causar dilatação dinâmica, sendo o exercício conhecido por exacerbar sintomas vagais preexistentes. Durante o exercício, o diâmetro da aorta ascendente aumenta aproximadamente $1 \mathrm{~mm}$ ou mais para cada aumento de $15 \mathrm{mmHg}$ na pressão sistólica ou diastólica, podendo causar uma compressão circunferencial no sistema respiratório de, aproximadamente, 3 a $6 \mathrm{~mm}^{1}$.

O processo de envelhecimento resulta na dilatação da aorta em, aproximadamente, $0,1 \mathrm{~cm}$ por década. Hipertensão e arteriosclerose podem dilatar e aumentar a tortuosidade da aorta, causando subsequente compressão traqueal. Esses dois mecanismos podem contribuir para o início dos sintomas no adulto. Finalmente, o processo de envelhecimento do tórax e das vértebras pode limitar as dimensões do mediastino, agravando a compressão ${ }^{1}$. A paciente relatada não apresenta, no presente momento, hipertensão arterial; no entanto, apresentava alterações degenerativas dos corpos vertebrais. 
A avaliação do paciente com suspeita de duplo arco aórtico inicia-se com a rotina do tórax: radiografia convencional em posição posteroanterior e perfil, podendo-se acrescentar o esofagograma como avaliação complementar ${ }^{1,9,11}$. Os sinais sugestivos de anel vascular são: alargamento do mediastino, presença de um botão aórtico à direita e evidência de compressão traqueal e/ou esofageana.

O ecocardiograma é útil na avaliação do arco aórtico e da anatomia do coração; no entanto, a TC e a ressonância magnética (RM) têm mostrado maior acurácia na avaliação das estruturas mediastinais ${ }^{1,9,11}$.

Nesse caso, a TC foi útil para o diagnóstico e delineamento da anatomia dos arcos, mostrando o dominante.

A Radiologia contribuiu muito para delinear as causas de obstrução das vias aéreas devido à compressão vascular, em 1940 com o emprego das radiografias convencionais e contrastadas, entre os anos de 1960-1970 com o advento da angiografia, e entre 1980-1990 com a TC e RM. Esses métodos de imagem facilitam o diagnóstico preciso e permitem um tratamento oportuno da doença ${ }^{11}$.

Concluindo, a compressão extrínseca devido ao anel vascular é extremamente rara, podendo ser silenciosa ou apresentar sintomas vagos ou exuberantes, tanto na infância quanto na idade adulta. Os meios de diagnóstico são complementares e fundamentais para se detectar a cardiopatia congênita.

\section{Referências}

1. Hardin RE, Brevetti GR, Sanusi M, et al. Treatment of symptomatic vascular rings in the elderly. Tex Heart Inst J. 2005;32:411-5.

2. Muto A, Nishibe T, Kondo Y, Sato M, Ando M. Detection and treatment of complete vascular ring in an elderly woman with dysphagia. J Thorac Cardiovasc Surg. 2005;130:585-6.
3. Lowe GM, Donaldson JS, Backer CL. Vascular rings: 10-year review of imaging. Radiographics. 1991;11:637-46.

4. Yedururi S, Guillerman RP, Chung T, et al. Multimodality imaging of tracheobronchial disorders in children. Radiographics. 2008;28:e29.

5. Longo-Santos LR, Maksoud-Filho JG, Tannuri U, et al. Vascular rings in childhood: diagnosis and treatment. J Pediatr (Rio J). 2002;78:244-50.

6. Backer CL, Mavroudis C, Rigsby CK, Holinger LD. Trends in vascular ring surgery. J Thorac Cardiovasc Surg. 2005;129:1339-47.

7. Backer CL, Mavroudis C. Congenital Heart Surgery Nomenclature and Database Project: vascular rings, tracheal stenosis, pectus excavatum. Ann Thorac Surg. 2000;69(4 Suppl):S308-18.

8. Mafeei FHA, Lastoria S, Yoshida WB, et al. Doenças Vasculares Periféricas: o desenvolvimento do sistema vascular. $3^{\mathrm{a}}$ ed. Rio de Janeiro: MEDSl; 2002. p. 3-17.

9. Harty MP, Kramer SS, Fellows KE. Current concepts on imaging of thoracic vascular abnormalities. Curr Opin Pediatr. 2000;12:194-202.

10. Knight L, Edwards JE. Right aortic arch. Types and associated cardiac anomalies. Circulation. 1974;50:1047-51.

11. Berdon WE. Rings, slings, and other things: vascular compression of the infant trachea updated from the midcentury to the millennium--the legacy of Robert E. Gross, MD, and Edward B. D. Neuhauser, MD. Radiology. 2000;216:624-32.

Correspondência:
Ana Rita Abrão
Avenida T-30, 1492, 20 andar - Setor Bueno
CEP 74215-060 - Goiânia (GO), Brasil
E-mail: abrao.ar@gmail.com
Contribuições dos autores:
Análise e interpretação dos dados: ARA, VQS, EOC
Coleta de dados: EOC
Redação do artigo: ARA, EOC
Revisão crítica do texto: ARA, EOC, RKB, ADM
Aprovação final do artigo*: ARA, VQS, EOC, RKB, ADM
Análise estatística: N/A
Responsabilidade geral pelo estudo: ARA, EOC, RKB, ADM
Tos os autores leram e aprovaram a versão final submetida ao J Vasc Bras.

